\title{
Innovación y competitividad: micro y pequeñas empresas del sector agroindustrial en Cúcuta
}

\author{
Innovation and competitiveness: micro and small enterprises of agroindustrial \\ sector in Cúcuta
}

Sharyn Nataly Hernández-Fuentes'
Karla Yohana Sánchez-Mojica ${ }^{2}$

Recibido: marzo 21 de 2017

Aceptado: junio 27 de 2017

\begin{abstract}
Resumen
La globalización es un factor importante en la toma de decisiones de países y empresas para entrar en la realidad donde la innovación es el principal componente para impulsar la competitividad. El objetivo de la investigación es analizar la innovación empresarial como eje de la competitividad para las microempresas y pequeñas empresas agroindustriales de Cúcuta. Se utiliza para efectos metodológicos un modelo de elección discreta sobre una muestra de empresas del sector agroindustrial. Esta investigación tiene un enfoque mixto, alcance descriptivo - explicativo, creando la necesidad de estudiar la generación de innovación empresarial. Los resultados muestran que las microempresas y pequeñas empresas del sector agroindustrial de Cúcuta deben mejorar su capacidad de innovar en proceso, producto y organización para ser más competitivos. Cúcuta, frontera y segunda ciudad con mayor desempleo en Colombia, necesita de la innovación empresarial para lograr competitividad y mejorar sus condiciones sociales desde lo empresarial.
\end{abstract}

Palabras Clave: agroindustria, competitividad, empresa, gestión, innovación.

\begin{abstract}
Globalization is an important factor in the decision-making of countries and companies to enter the reality where innovation is the main component to boost competitiveness. The objective of the research is to analyze business innovation as the axis of competitiveness for micro-enterprises and small agro-industrial companies in Cúcuta. A discrete choice model for a sample of companies in the agro-industrial sector is used for methodological purposes. This research has a mixed approach: descriptive - explanatory scope, creating the need to study the generation of business innovation. The results show that micro-enterprises and small companies in the agro-industrial sector of Cúcuta should improve their capacity to innovate in process, product and organization to be more competitive. Cúcuta, the frontier and second most unemployed city in Colombia, needs business innovation to achieve competitiveness and improve its social conditions from the business point of view.
\end{abstract}

Keywords: agro-business, competitiveness, business, management, innovation.

\footnotetext{
1 Economista, Especialista en Gerencia Financiera, Fundación de Estudios Superiores Comfanorte, Colombia. E-mail: sn_hernandez@ fesc.edu.co

2 Ingeniera en Telecomunicaciones, Especialista en Docencia Universitaria, Fundación de Estudios Superiores Comfanorte, Colombia. E-mail: investigaciones@fesc.edu.co
} 


\section{Introducción}

El mundo actual está en constante evolución. La globalización es un factor importante en la toma de decisiones de países y empresas, que buscan entrar en esta nueva realidad donde no existen las fronteras geográficas, culturales, ni económicas o empresariales. Los adelantos tecnológicos y la evolución constante suponen un avance en la gestión empresarial, logrando la innovación a grandes escalas, porque como lo asegura el ex presidente de República Dominicana Leonel Fernández (2014, p. 2): "Lo que se procura, por vez primera en la historia de la humanidad, es poder producir cualquier producto en cualquier lugar del mundo, y que pueda ser consumido por cualquier persona en cualquier otro lugar del globo".

En las empresas afecta cuando se dedican a la cooperación competitiva con el fin de innovar su modelo de negocio; una investigación realizada por Chander (2016), muestra que en mercados de redes, cuando las empresas optan por participar en la cooperación competitiva a la luz de la amenaza competitiva que se hace para adoptar una estrategia defensiva u ofensiva, se ven obligadas a generar cambios y desconocen que las mejoras en producto o procesos se denomina innovación, siendo este concepto poco entendido y valorado por el empresario.

Es importante que las personas en una empresa comprendan que los resultados también dependen del grado de innovación de los procesos que lideran e influyen positivamente en el rendimiento del negocio (Rangus, \& Slavec, 2017). Francia, Broman, Robert, Basile y Trygg (2017) en su investigación afirman que el negocio exitoso es cada vez más acerca de la comprensión de los desafíos y oportunidades vinculados a la transición de la sociedad hacia la sostenibilidad y, por ejemplo, ser capaz de innovar, diseñar y construir modelos de negocio que son funcionales en este contexto.

En el caso de las empresas agroindustriales, estas cuentan con un componente que no se puede de- jar de lado en la ejecución de planes de innovación, siendo la naturaleza su principal aliado para la producción de materias primas (Delgado, 2016). En este sentido Herrera (2016), expone que las empresas pueden lograr un impacto social duradero apuntando a factores sistémicos, utilizando la ecología para el crecimiento inclusivo, caso importante en la agroindustria de cualquier país.

Prajogo (2016), utilizando los datos de 207 empresas de fabricación en Australia, muestra que los entornos dinámicos refuerzan el efecto de la innovación de productos en el rendimiento del negocio. Este artículo examina los entornos del rol del negocio (en términos de dinamismo y competitividad) como factores de contingencia que afectan a la eficacia de los diferentes tipos de estrategias de innovación (en términos de producto y de proceso) en la entrega de los resultados empresariales.

Las investigaciones recientes y la práctica demuestran que la innovación modelo de negocio es un enfoque prometedor para la mejora de la sostenibilidad en las empresas (Yang, Evans, Vladimirova, \& Rana, 2017). Un ejemplo de ello es el trabajo de Gupta, Malhotra, Czinkota y Foroudi (2016), donde emplean la teoría de la complejidad para investigar la relación entre la competitividad y la innovación en las prácticas de márqueting de las grandes empresas manufactureras como revendedores de su marca. Este estudio revela que la capacidad de innovación de las iniciativas mejora la competitividad de las empresas reflejadas en las ventas y exportaciones de las empresas en estudio. Lo anterior es una evidencia de la relación directa entre innovación y competitividad en busca de valores agregados, junto con mayor posicionamiento en el mercado.

El sector agroindustrial, de manejo directo de recursos naturales, debe según Rosca, Arnold y Bendul (2016), tener en cuenta que las innovaciones sostenibles son invenciones que proporcionan un progreso esencial relativo a los problemas sociales, económicos y ecológicos. Las empresas de este sector están impactando directamente la so- 
ciedad, la economía de un país, la economía del mundo y el ambiente con buenas prácticas de producción. Generando esas buenas prácticas es donde se mejoran los procesos con el objetivo de obtener mayor calidad en servicios o productos, siendo el camino más adecuado la innovación (Bolton, \& Hannon, 2016).

En algunos países la innovación en las empresas sigue desempeñando un papel fundamental en la mejora del rendimiento y en el mantenimiento de ventajas competitivas en los mercados globales. En el caso específico de la industria agroalimentaria, es notorio como las innovaciones se han convertido en un instrumento importante para las empresas de este sector (Zouaghi, \& Sánchez, 2016).

En Colombia, la innovación empresarial ha sido uno de los aspectos a mejorar desde hace ya algunas décadas. De acuerdo con el portal interactivo que ofrece Portafolio.co denominado Emprendimiento Colombiano en Cifras, para el año 2016, Colombia pasó del puesto 60 al 68 en el Índice de Innovación Global (GII), siendo Suiza el primer lugar en el ranking donde se evalúan 143 países.

El sector agroindustrial colombiano presenta poca innovación y dentro de los tipos de innovación no se evidencian grandes adelantos en la aplicación de tecnología, es decir que la innovación tecnológica es escasa (Becerra-Gualdrón, \& Cruz-Vásquez, 2014). Situación similar se presenta en Chile, según investigación realizada por Geldes, Felzensztein y Fenech (2016), quienes muestran que sólo las innovaciones de los productos afectan significativamente el rendimiento de la innovación en todas las industrias. Adicionalmente, las innovaciones tecnológicas y no tecnológicas afectan de manera diferente a las empresas. Las innovaciones tecnológicas muestran un mayor impacto en el mejoramiento y tecnificación de procesos o productos terminados (Ince, Imamoglu, \& Turkcan, 2016).

El impacto del emprendimiento y la innovación sobre los negocios avanza de una manera tan rápi- da y tiene un alcance tan grande, que resulta difícil entender la manera adecuada de proceder para lograr los resultados que un país como Colombia está buscando en este ámbito. Lo que está menos claro es la manera en la que pueden llegar a ese estado las naciones que, a diferencia de los desarrollados, aún están lejos de este emprendimiento innovador (Valdelamar-Zapata, Ramírez-Cruz, Rodríguez-Rivera, \& Morales-Rubiano, 2015).

La innovación determina cada vez más el crecimiento sostenido de las empresas. Éstas invierten en innovación porque la innovación representa la mejor oportunidad para aumentar cuota de mercado, reducir costes de producción y por ende incrementar los beneficios, lo que se traduce en mayor competitividad (Pertúz-Peralta, \& Perez-Orozco, 2016; Hernández-Taboada, Bustamante-Zapata, \& Porto-Pérez, 2014).

La competitividad local significa la capacidad de las empresas de un país, o de una región específica, para diseñar, desarrollar, producir y colocar sus productos en el mercado internacional en medio de la competencia con empresas de otros países (Norton, 2017). El aprovechamiento de los recursos locales endógenos, tanto culturales, institucionales, económicos, sociales, ambientales y políticos es de vital importancia para el desarrollo de sistemas territoriales innovadores y competitivos (González-Calixto, Hernández-Plazas, \& Corredor-Balaguera, 2013).

Es claro que los efectos de la innovación del modelo de negocio y la innovación concepto de negocio son una relación actual que no debe desligarse (Ruta N Medellín, 2016). La adopción de nuevos modelos y conceptos que apoyan la innovación es importante; las claves de innovaciones incrementales y radicales exitosas se encuentran en la adopción de un nuevo marco contextual y conceptual, a través del cual pueden producirse innovaciones y las necesidades del cliente pueden ser satisfechas, lo que a su vez genera nuevas ventajas competitivas (Souto, 2015). 
La ciudad de Cúcuta (Colombia), golpeada fuertemente por decisiones gubernamentales de Colombia y Venezuela, que la posicionan como la segunda ciudad a nivel nacional con mayor tasa de desempleo del $14.9 \%$, es superada sólo por Quibdó con un 17,8\% (Filco, 2016).

En Cúcuta, de acuerdo con el informe de gestión de la Cámara de Comercio de Cúcuta, CCC, para el año 2011 habían registradas 23.946 empresas, de las cuales el $95,36 \%$ son Microempresas - es decir, $22.837-$; $3,68 \%$ son pequeñas empresas; $0,58 \%$ son medianas y sólo el $0,36 \%$ son grandes empresas, las cuales corresponden únicamente a 88 empresas (Cámara de Comercio de Cúcuta, 2012). Además, para el año 2014, el tejido empresarial del municipio presentaba una estructura por número de unidades económicas así: 52,2\% en comercio, $36,2 \%$ en servicios, $10 \%$ en industria y $1,2 \%$ en otras actividades económicas (Data Cúcuta, 2014). Es decir que cerca del $88 \%$ de las empresas de Cúcuta pertenecen al sector terciario y de servicios, lo cual es aún más preocupante considerando que la industria, que es el sector con mayor generación de empleo y mayor crecimiento de la economía, está presente en la Ciudad con solo una participación del $10 \%$.

Esta investigación tiene como objetivo analizar el proceso de innovación empresarial como eje principal de la competitividad local, específicamente en la ciudad de Cúcuta, evaluando una muestra de las micro y pequeñas empresas del sector agroindustrial bajo un instrumento validado que permita hacer un diagnóstico del nivel de innovación y detectar mejoras en la situación laboral, social y económica que se presenta actualmente en la ciudad.

\section{Materiales y Métodos}

El enfoque de la investigación es mixto porque parte del análisis cualitativo de variables endógenas, que por revisión bibliográfica tienen incidencia en la innovación de las micro y pequeñas empresas industriales. De igual forma, contempla un procedimiento estadístico producto de la aplicación de un instrumento que permite obtener información primaria de la población objetivo, generando un análisis cuantitativo. Además, se contempla desde un alcance descriptivo - explicativo creando la necesidad de estudiar la generación de innovación empresarial.

Para la aplicación del instrumento de investigación, después de ser validado por medio de expertos, se solicitó a la Cámara de Comercio de Cúcuta la información de la población de micro y pequeñas empresas agroindustriales registradas, siendo la base de datos de 49 empresas. A través del muestreo aleatorio simple no estratificado, se calculó la muestra con la fórmula de población finita, asignando un error máximo del $6 \%$ con una probabilidad del 0.5 , estableciendo una muestra final de 44 empresas agroindustriales a las cuales se aplicó el instrumento. Las micro y pequeñas empresas representan un $98 \%$ del total de las empresas en la ciudad. Se toma como referencia el sector Agroindustrial debido a que es el principal generador de cambio y crecimiento económico en la región, además es un sector económico priorizado por el plan estratégico del departamento de Norte de Santander (Castillo, 2016).

La investigación inicia indagando sobre la capacidad de innovación empresarial actual en las micro y pequeñas empresas agroindustriales de Cúcuta. Posteriormente, se determina la relación existente entre la innovación empresarial y el aumento de la competitividad local. Por último, se establecen parámetros que permitan a las empresas autoevaluarse consiguiendo lineamientos sobre la forma adecuada para avanzar e innovar.

La encuesta se elaboró siguiendo los lineamientos de Alderete y Diez (2014). Las primeras 3 preguntas buscan definir el grado de innovación, diferenciando en el producto, en el proceso productivo y en la organización. Luego, con una pregunta se busca definir la clasificación de empresas que más generan innovación en Cúcuta. Se continúa con 3 preguntas que buscan determinar las condiciones que propician la innovación mediante estra- 
tegias de negociación con proveedores, vínculos con asociaciones o gremios y asociaciones entre empresas. Por último, 3 preguntas que definen el perfil innovador de las empresas considerando la inversión en maquinaria y equipos, el acceso a las Tecnologías de la Información y la Comunicación, TIC, y la contratación de sus empleados por nivel educativo.

\section{Resultados y discusión}

En primer lugar se realizó una revisión bibliográfica que permitió determinar la relación entre innovación y competitividad. El desarrollo de este objetivo permite evidenciar la relación existente entre la innovación y la competitividad de las empresas frente a otras del mismo sector o de diferente municipio.

En Colombia, de acuerdo con el Ministerio de Tecnologías de la Información y Comunicaciones, MinTIC, a través de la iniciativa de Investigación, Desarrollo e Innovación, I+D+i, se promueven los Nodos de Innovación, con la participación activa de entidades del Gobierno, entidades del sector productivo/industrial TIC y la Academia. Lo que hace el MinTIC es conformar bancos de proyectos elegibles para la generación de soluciones y productos a los cuales las entidades del Estado puedan acceder en el mercado, alineados con las prioridades establecidas por los Nodos de Innovación en documentos denominados Agendas Estratégicas de Innovación, AEl, todo con el fin de mejorar la competitividad empresarial en cada uno de los departamentos del país.

Las empresas que más innovan pueden ser las más competitivas porque están a la vanguardia de los procesos y técnicas para desarrollar de manera eficiente su actividad productiva. La relación que existe entre la innovación y la competitividad es directa y positiva, porque a medida que se incremente la innovación en las empresas también se incrementa la competitividad frente a otras empresas del mismo sector, o frente a conglomerados de pequeñas empresas que siguen manteniendo su labor de manera rudimentaria. La innovación parte desde el uso de tecnologías, hasta la reorganización de la empresa en áreas administrativas y operativas a fin de establecer la importancia de la mejora continua. La competitividad se ve reflejada en el aumento de las ventas, de los ingresos y por ende de los beneficios percibidos (Servicio Nacional de Aprendizaje, 2001).

Tomando como referencia a Castro y Feria (2012), quienes desarrollan un análisis sobre la competitividad de las empresas en Aguascalientes México, la presente investigación realiza una propuesta de autoevaluación empresarial a partir de una investigación interna de la organización, como se observa en las figuras 1 y 2 . Estas características se identifican a partir de la revisión bibliográfica realizada por la autora, y a partir de deducciones obtenidas mediante la respuesta en la encuesta aplicada.

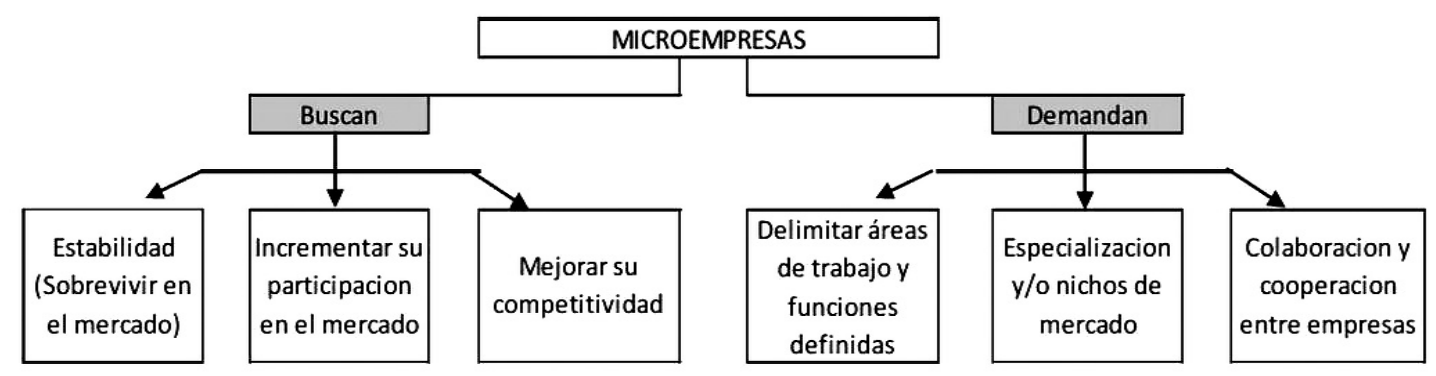

Figura 1. Características para evaluación en micro empresas. 


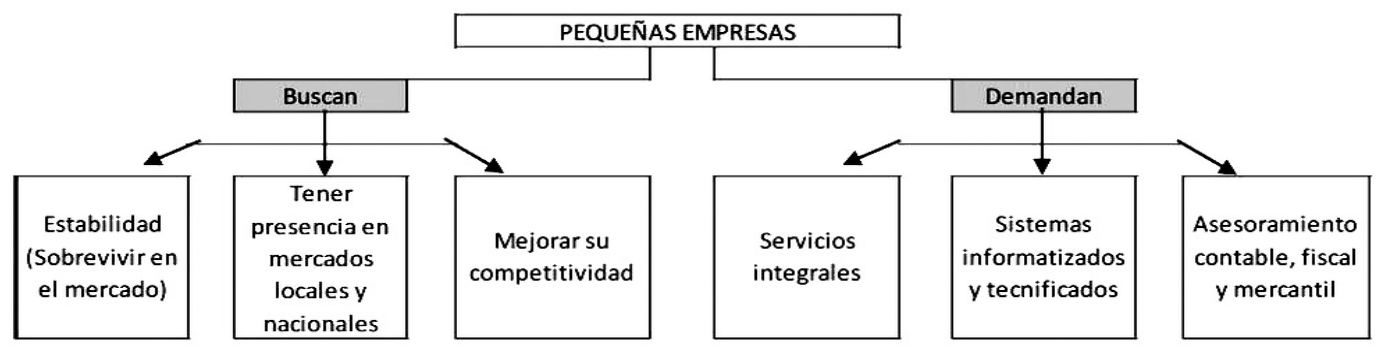

Figura 2. Características para evaluación en pequeñas empresas.

Las figuras 1 y 2 permiten establecer que el proceso de evaluación en las microempresas y en las pequeñas empresas, toman en cuenta factores como: participación en el mercado, competitividad y estabilidad. Además, las necesidades de las empresas varían dependiendo de su clasificación, siendo éste un factor importante para esta investigación a la hora de proponer y recomendar medidas que impulsen la innovación. Básicamente se establecen 3 factores importantes de los cuales se desprende el análisis: Talento Humano, Inversión en maquinaria y equipo, y Vinculación del sector académico; cada factor tiene sus características, las cuales deben estar presentes en la empresa para lograr diferentes resultados en cada factor.

Luego de aplicar el instrumento de investigación, en los resultados tabulados y analizados se encuentra que un $91 \%$ de los encuestados realizó alguna innovación en su producto. Sin embargo, es muy reducido el porcentaje de empresas que incorpora un nuevo producto al mercado (solo el $2 \%)$, y las demás solo han variado el envase, el sabor o los insumos utilizados. El $11 \%$ de las empresas entrevistadas no realizó ninguna innovación, lo que evidencia falta de orientación sobre su aplicación.

También se evidenció que el $25 \%$ de las empresas no realizó innovación en el proceso productivo y solo un $7 \%$ estableció un nuevo proceso en el mercado. Según el modelo que guía la aplicación del instrumento, las empresas que no realizan mejoras en los procesos productivos tienden a desaparecer en un tiempo aproximado de 4 a 5 años, por los cambios constantes del mercado nacional o internacional. Adicionalmente, el $63 \%$ de las empresas encuestadas no realizaron ninguna innovación en la organización de la empresa, ni en sus procesos administrativos. Solo el $7 \%$ realizó una reorganización de procesos y actividades administrativas, considerando las capacidades de los trabajadores.

Vale la pena resaltar que la inversión en innovación resulta importante a la hora de mejorar la capacidad productiva y el posicionamiento de una empresa en el mercado. Este aspecto se destaca en la investigación de Cote-Peña, Meneses-Amaya, Arenas-Morantes, y Caballero-Pérez (2016) donde se asegura que para perfilarse como una región empresarial altamente competitiva, es necesario mejorar los niveles de inversión en actividades científico-tecnológicas que incrementen las innovaciones de las empresas, demostrando que en Antioquia es mayor el porcentaje de inversión por parte de las empresas en Actividades de Ciencia, Tecnología e Innovación (ACTI), a diferencia del departamento de Santander.

Así mismo, es necesario percibir la capacitación de los empleados como una inversión y no como un gasto. En este sentido, Parra-Penagos y Rodríguez-Fonseca (2015), aseguran que es muy importante la capacitación a los empleados porque integra la organización gracias al diseño de planes como estrategia para el crecimiento y la competitividad, considerando a su vez que representa una inversión a corto y largo plazo benéfica para la organización y el empleado. 
La aplicación del instrumento también demuestra una relación entre el nivel de innovación y el tamaño de la empresa. Tomando en cuenta lo indicado por el artículo 43 de la Ley 1450 de 2011, los parámetros vigentes para clasificar las empresas son el número de empleados que tenga la empresa, y la cantidad de activos, demostrando que de las 44 encuestas realizadas, 35 son microempresas (de 1 a 10 trabajadores) es decir el $79,5 \%$ y solo 9 empresas son pequeñas (de 11 a 50 trabajadores) es decir, el 20,4\%. De igual forma, el $48 \%$ de las empresas encuestadas no tiene acuerdos comerciales. Sin embargo, cabe aclarar que todas las empresas pequeñas que fueron encuestadas tienen acuerdos entre empresas del mismo sector o acuerdos intersectoriales que buscan su fortalecimiento, lo cual indica que son las microempresas quienes no poseen estos acuerdos.

En cuanto al interés por realizar alianzas estratégicas que permitan mejorar la capacidad productiva y la generación de negocio a través de las iniciativas de asociación, se concluye que el $70 \%$ si realiza asociaciones con otras empresas y se cooperan. El hecho de pertenecer a las agremiaciones puede permitir un mejoramiento continuo considerando la interacción entre empresarios de un mismo sector, que sufren y padecen situaciones similares. Esto permite ver la relación entre la innovación a partir de la participación activa dentro de las agremiaciones. Se evidencia que solo el $18 \%$ mantiene una relación continua con la agremiación.

También se evidenció que el $46 \%$ realiza inversión sólo cuando es necesario y únicamente el $16 \%$ de las empresas realiza inversión anual en maquinaria y equipos. En esta pregunta se les explicaba a los encuestados que las TIC hacen referencia a las tecnologías de la información y comunicaciones, dando como resultado que el $82 \%$ utiliza tecnología dentro del desarrollo habitual de su proceso productivo, mientras que solo un $2 \%$ no tiene acceso a ella y realiza sus actividades de manera artesanal.

En la tabla 1 se muestra la realidad de la contratación en las micro y pequeñas empresas agroindustriales de Cúcuta, como referente importante para medir la innovación empresarial desde los procesos gerenciales.

\begin{tabular}{|c|c|c|c|}
\hline \multicolumn{4}{|c|}{ Número de empleados con título profesional en las empresas } \\
\hline & De 1 a 5 & 28 & $64 \%$ \\
\hline \multirow[t]{2}{*}{$\mathrm{SI}$} & De 6 a 10 & 2 & $5 \%$ \\
\hline & Más de 10 & 0 & $0 \%$ \\
\hline \multicolumn{2}{|l|}{ No tiene } & 14 & $31 \%$ \\
\hline \multicolumn{2}{|l|}{ TOTAL } & 44 & $100 \%$ \\
\hline \multicolumn{4}{|c|}{ Número de empleados con nivel técnico o tecnólogo en las empresa } \\
\hline & De 1 a 5 & 2 & $5 \%$ \\
\hline \multirow[t]{2}{*}{$\mathrm{SI}$} & De 6 a 10 & 25 & $56 \%$ \\
\hline & Más de 10 & 6 & $14 \%$ \\
\hline \multicolumn{2}{|l|}{ No tiene } & 11 & $25 \%$ \\
\hline \multicolumn{2}{|l|}{ TOTAL } & 44 & $100 \%$ \\
\hline \multicolumn{4}{|c|}{ Número de empleados con bachillerato en las empresas } \\
\hline & De 1 a 5 & 1 & $2 \%$ \\
\hline \multirow[t]{2}{*}{$\mathrm{SI}$} & De 6 a 10 & 26 & $59 \%$ \\
\hline & Más de 10 & 17 & $39 \%$ \\
\hline \multicolumn{2}{|l|}{ No tiene } & 0 & $0 \%$ \\
\hline \multicolumn{2}{|l|}{ TOTAL } & 44 & $100 \%$ \\
\hline
\end{tabular}

Tabla 1. Contratación según cualificación. 
En el análisis de la tabla 1 se puede identificar que existe un $31 \%$ del total de las empresas que no cuenta dentro de su planta con trabajadores profesionales; además, un $25 \%$ de las empresas no tienen trabajadores con nivel académico técnico o tecnológico. Todas las empresas cuentan con trabajadores bachilleres, y el 59\% de las empresas asegura que tiene en su planta de personal entre 6 y 10 bachilleres, lo cual resulta interesante de evaluar, considerando que por su clasificación las microempresas tienen una planta de personal no su- perior a 10 empleados, mientras que las pequeñas empresas tienen una planta de personal de entre 11 y 50 trabajadores, según la Ley 905 de 2004.

En cuanto al tercer objetivo específico, en la figura 3 se presenta una propuesta de autoevaluación para las empresas, a fin de que puedan verificar los requisitos mínimos que permitan lograr la generación de un sistema de innovación empresarial, que obtenga como resultado la competitividad local.

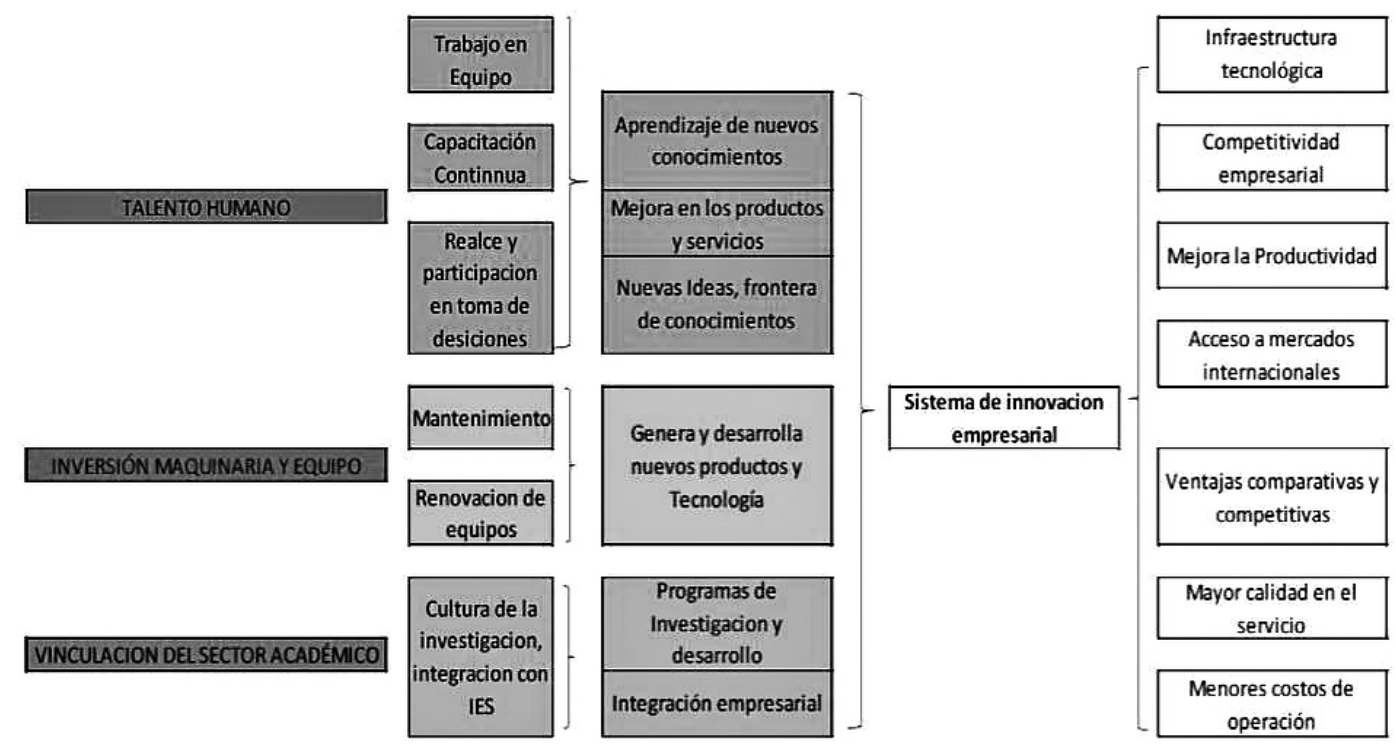

Figura 3. Propuesta para la evaluación de la innovación empresarial.

La propuesta surge de la revisión bibliográfica y se valida con los resultados de la aplicación del instrumento de investigación. Básicamente se establecen 3 factores importantes de los cuales se desprende el análisis: Talento Humano, Inversión en maquinaria y equipo, y Vinculación del sector académico. Cada uno de ellos tiene sus características, las cuales deben estar presentes en la empresa para lograr diferentes resultados por cada factor.

En el modelo se definen las características de manera diferenciada, considerando por separado las microempresas (o empresas familiares), por su complejidad, ambiente emocional de crisis y conflictos familiares; y las pequeñas empresas que tienen mayor producción y procesos diferentes. Lo anterior conlleva a la generación de un sistema de innovación empresarial, el cual, aplicado correctamente obtiene resultados como infraestructura tecnológica, la competitividad empresarial, mejora de la productividad, acceso a mercados internacionales, ventajas comparativas y competitivas, mayor calidad en el servicio prestado y menores costos de operación.

Los resultados presentan una relación importante con los hallazgos de Bolton y Hannon (2016), 
quienes muestran la importancia de la innovación empresarial a través del uso de la tecnología, criterio que se evidencia como debilidad en las empresas aquí evaluadas. Los autores muestran un ejemplo de modelo de negocio innovador en la transformación de los sistemas socio-técnicos y se centran en las tecnologías de energía descentralizadas, explorando la innovación del modelo de negocio en el contexto de una transición hacia un sistema económicamente sostenible y aplicable a cualquier empresa.

Así mismo, se evidenció en la presente investigación que la voluntad de implementar procesos de innovación empresarial está directamente relacionada con un mejor posicionamiento en el mercado. Esto coincide con el trabajo de Rusu (2016), donde se concluye que es de gran importancia la decisión del empresario de colocar en práctica, por un periodo corto de tiempo, una innovación en el producto, en el proceso, la comercialización y la organización empresarial, evidenciando el impacto y transformación de la empresa debido a los cambios realizados.

\section{Conclusiones}

Las microempresas y pequeñas empresas del sector agroindustrial de Cúcuta evidencian que falta mejorar su capacidad de innovar en el proceso, producto y organización empresarial a fin de contribuir a la competitividad local de la ciudad. Esto se soporta en que el $11 \%$ de las empresas encuestadas no realizó ninguna innovación en los últimos 5 años, el $48 \%$ no tiene ningún acuerdo comercial con otras empresas y el $32 \%$ no tiene contacto con las agremiaciones empresariales.

La innovación tiene una relación directa y positiva con la competitividad, debido a que no se puede ser competitivo frente a otras empresas si no se tienen mejoras atractivas dentro del proceso productivo, en el producto o servicio, o en la organización. La innovación no debe ser necesariamen- te algo extraordinariamente nuevo; pero sí que marque la diferencia y permita la fidelización del cliente.

En este sentido se presentó una propuesta de autoevaluación empresarial que permita verificar efectivamente las condiciones internas de la empresa para la generación de un sistema de innovación. Este sistema permitirá obtener resultados como infraestructura tecnológica, la competitividad empresarial, mejora de la productividad, acceso a mercados internacionales, ventajas comparativas y competitivas, mayor calidad en el servicio prestado y menores costos de operación. La propuesta se plantea con el fin de que las empresas consideren los factores expuestos y puedan autoevaluarse, consiguiendo generar una innovación que propicie la competitividad local en las empresas agroindustriales de Cúcuta.

\section{Referencias}

Alderete, M. V., \& Diez, J. I. (2014). Innovación para la competitividad territorial: un análisis de las pymes agroindustriales de Bahía Blanca. Revista Líder, 25, 35-69.

Becerra-Gualdrón, C. J., \& Cruz-Vásquez, J. L. (2014). Diagnóstico de la competitividad agroindustrial en el departamento de Boyacá para el año 2011. Revista de Investigación, Desarrollo e Innovación, 4 (2), 111-123. Doi: http://dx.doi. org/10.19053/20278306.2961

Bolton, R., \& Hannon, M. (2016). Governing sustainability transitions through business model innovation: Towards a systems understanding. Research Policy, 45 (9), 1731-1742. doi: https://doi. org/10.1016/j.respol.2016.05.003

Cámara de Comercio de Cúcuta (2012). Informe de gestión año 2012. Recuperado de: http://www.cccucuta.org.co/media/Documentos/informe_de_ gestion_2012.pdf 
Castillo, O. R. (2016). Turismo, arcilla y agroindustria, tres líneas priorizadas para el proyecto Diamante Caribe y Santanderes. Oficina de prensa Gobernación de Norte de Santander. Recuperado de: http://www.nortedesantander.gov.co/Noticias-Gobernaci\%C3\%B3n-Norte-de-Santander/ ArticleID/4401/diamante

Castro, J., \& Feria, M. (2012). La innovación empresarial: una propuesta de investigación-consultoría para la competitividad en aguas calientes, México. Revista científica Teorías, Enfoques y Aplicaciones en las ciencias sociales. Recuperado de: http://132.248.9.34/hevila/Revistacientificateoriasenfoquesyaplicacionesenlascienciassociales/2012/no9/5.pdf

Cote-Peña, C., Meneses-Amaya, C., Arenas-Morantes, C., \& Caballero-Pérez, D. (2016). Benchmarking entre sistemas regionales de innovación: el caso de Santander y Antioquia, Colombia. Revista de Investigación, Desarrollo e Innovación, 7 (1), 11-24. doi: http://dx.doi.org/10.19053/20278306. v7.n1.2016.4088

Data Cúcuta: Observatorio Económico de la Cámara de Comercio de Cúcuta (Mayo de 2014). Caracterización del Tejido Empresarial de Cúcuta. Recuperado de: http://www.datacucuta.com/PDF/ estudios-sectoriales/5.EMPRESARIAL/tejido\%20 empresarial.pdf

Delgado, B. (2016). Tecnología y naturaleza, aliados de la agricultura inteligente para adaptarse al clima. Revista 20 minutos. Recuperado de: http:// www.20minutos.es/noticia/2774252/0/agricultura-tecnologia-naturaleza-clima/

Fernández, L. (2014). La globalización y los TLC. Republica Dominicana: Instituto Internacional de Diseño.

Fuente de Información Laboral en Colombia. FILCO. (Junio de 2016). Boletín de Análisis Coyuntural La- boral. Recuperado de: http://filco.mintrabajo.gov. co/pages/panoramaMundoLaboral.xhtml

Francia, C. L., Broman, G., Robert, K. H., Basile, G., \& Trygg, L. (2017). An approach to business model innovation and design for strategic sustainable development. Journal of Cleaner Production, 155166.

Geldes, C., Felzensztein, C., \& Fenech, J. P. (2016). Technological and non-technological innovations, performance and propensity to innovate across industries: The case of an emerging economy. Industrial Marketing Management, 61, 55-56. doi: https://doi.org/10.1016/j.indmarman.2016.10.010

González-Calixto, M. B., Hernández-Plazas, V. M., \& Corredor-Balaguera, L. M. (2013). El diseño organizacional como estrategia de fortalecimiento para la empresa Calcalizas de Nobsa, Boyacá. Revista de Investigación, Desarrollo e Innovación, 3(2), 87-100. doi: $10.19053 / 20278306.2170$

Gupta, S., Malhotra, N. K., Czinkota, M., \& Foroudi, P. (2016). Marketing innovation: A consequence of competitiveness. Journal of Business Research, 69 (12), 5671-5681. doi: https://doi.org/10.1016/j. jbusres.2016.02.042

Hernández-Taboada, F. D., Bustamante-Zapata, L. F., \& Porto-Pérez, I. A. (2014). Evaluación del grado de competitividad de cinco empresas del sector lechero de Sincelejo, Colombia. Revista de Investigación, Desarrollo e Innovación, 5 (1), 8-19. doi: https://doi.org/10.19053/20278306.3135

Herrera, M. E. (2016). Innovation for impact: Business innovation for inclusive growth. Journal of Business Research, 69, (5) 1725-1730.

Ince, H., Imamoglu, S. Z., \& Turkcan, H. (2016). The Effect of Technological Innovation Capabilities and Absorptive Capacity on Firm Innovativeness: A Conceptual Framework. Procedia - Social and 
Behavioral ciences, 235, 764-770. doi: https://doi. org/10.1016/j.sbspro.2016.11.078

Norton, R. D. (2017). Colombia: Una evaluación estratégica de la Nacional de Cultivos de Competitividad. La Competitividad de la Agricultura Tropical, 87-145.

Parra-Penagos, C., \& Rodríguez-Fonseca, F. (2016). La capacitación y su efecto en la calidad dentro de las organizaciones. Revista de Investigación, Desarrollo e Innovación, 6 (2), 131-146. doi: http://dx. doi.org/10.19053/20278306.4602

Pertuz-Peralta, V., \& Perez-Orozco, A. (2016). Modelo de cultura organizacional innovadora en caficultores del departamento del Cesar, Colombia. Revista de Investigación, Desarrollo e Innovación, 6(2), 117130. doi:https://doi.org/10.19053/20278306.3687

Prajogo, D. I. (2016). The strategic fit between innovation strategies and business environment in delivering business performance. International Journal of Production Economics, 171, 241-249. doi: https://doi.org/10.1016/j.ijpe.2015.07.037

Rangus, K., \& Slavec, A. (2017). The interplay of decentralization, employee involvement and absorptive capacity on firms' innovation and business performance. Technological Forecasting and Social Change, Online, 120 (c) 195-203.

Rosca, E., Arnold, M., \& Bendul, J. C. (2016). Business models for sustainable innovation - an empirical analysis of frugal products and services. Journal of Cleaner Production. doi: https://doi.org/10.1016/j. jclepro.2016.02.050

Rusu, B. (2016). The Impact of Innovations on the Business Model: Exploratory Analysis of a Small Travel Agency. Procedia - Social and Behavioral Sci- ences, 221, 166 - 175. doi: https://doi.org/10.1016/j. sbspro.2016.05.103

Ruta N Medellín. (2016). Innovación en Modelos de Negocio. Recuperado de: http://www.rutanmedeIlin.org/es/recursos/abc-de-la-innovacion/item/ innovacion-en-modelos-de-negocio

Servicio Nacional de Aprendizaje. (2001). Política Nacional para la productividad y competitividad. Banco de la República. Recuperado de: http:// www.banrepcultural.org/node/69886

Souto, J. E. (2015). Business model innovation and business concept innovation as the context of incremental innovation and radical innovation. Tourism Management, 51, 142-155.

Valdelamar-Zapata, J. A., Ramírez-Cruz, Y. L., Rodríguez-Rivera, P. D., \& Morales-Rubiano, M. E. (2015). Capacidad innovadora: cómo fomentarla, según docentes de Ciencias Económicas e Ingeniería de la UMNG. Revista de Investigación, Desarrollo e Innovación, 6 (1), 7-14. doi: http://doi. org/10.19053/20278306.3454

Velu, C. (2016). Evolutionary or revolutionary business model innovation through coopetition? The role of dominance in network markets. Industrial Marketing Management, 124-135.

Yang, M., Evans, S., Vladimirova, D., \& Rana, P. (2017). Value uncaptured perspective for sustainable business model innovation. Journal of Cleaner Production, 1794-1804.

Zouaghi, F., \& Sánchez, M. (2016). Has the global financial crisis had different effects on innovation performance in the agri-food sector by comparison to the rest of the economy?. Trends in Food Science \& Technology, 50, 230-242. 